\title{
Structural and paramagnetic behavior of spinel NiCr2O4 nanoparticles synthesized by thermal treatment method: effect of calcination temperature
}

\begin{abstract}
Spinel nickel chromite nanoparticles were synthesized using a simple thermal treatment method. The effect of calcination temperatures on the final properties of obtained materials was carefully examined using various characterization techniques. The infrared spectra of nickel chromite $(\mathrm{NiCr} 2 \mathrm{O} 4)$ revealed the characteristic bonds of metalï oxygen for Nisingle bond $\mathrm{O}$ and $\mathrm{Crsingle}$ bond $\mathrm{O}$ bands around 600 and $470 \mathrm{cmi} 1$, respectively. The powder X-ray diffraction patterns exhibited the formation of normal spinel phase of $\mathrm{NiCr} 2 \mathrm{O} 4$ in the calcination process at temperature between 550 and $850{ }^{\circ} \mathrm{C}$. From transmission electron micrographs, nanosized particles with average size of $~ 7 і ̈ 64 \mathrm{~nm}$ were observed at calcination temperatures of $5501850{ }^{\circ} \mathrm{C}$, respectively. The calcined samples at 750 and $850{ }^{\circ} \mathrm{C}$ exhibited paramagnetic behavior with g-factor values of 1.92 and 2.15 , peak-to-peak line width of 25.59 and 117.02 Oe and resonance magnetic field of 342.04 and 306.49 Oe, respectively. Variation in the value of g-factor, peak-to-peak line width and resonance magnetic field can be attributed to the dipoleï dipole and super exchange interactions.
\end{abstract}

Keyword: A. Nanostructures; C. Electron microscopy; C. Infrared spectroscopy; C. X-ray diffraction 